\title{
Thermal Infrared Image Processing to Assess Heat Generated by Magnetic Nanoparticles for Hyperthermia Applications
}

\author{
Raquel O. Rodrigues ${ }^{1}$, Helder T. Gomes ${ }^{1}$, Rui Lima ${ }^{2,3,4}$, Adrián M.T. Silva ${ }^{5}$, Pedro \\ J.S. Rodrigues ${ }^{2}$, Pedro B. Tavares ${ }^{6}$, João Manuel R.S. Tavares ${ }^{7, *}$ \\ ${ }^{1}$ LCM - Laboratory of Catalysis and Materials - Associate Laboratory LSRE-LCM, Poly- \\ technic Institute of Bragança, Bragança, Portugal \\ ${ }^{2}$ Polytechnic Institute of Bragança, Bragança, Portugal \\ ${ }^{3}$ University of Minho, Mechanical Engineering Department, Guimarães, Portugal \\ ${ }^{4}$ CEFT, Faculdade de Engenharia, Universidade do Porto, Porto, Portugal \\ ${ }^{5}$ LCM - Laboratory of Catalysis and Materials - Associate Laboratory LSRE-LCM, Facul- \\ dade de Engenharia, Universidade do Porto, Porto, Portugal \\ ${ }^{6}$ CQVR, Chemical Department, Universidade de Trás-os-Montes e Alto Douro, Vila Real, \\ Portugal \\ ${ }^{7}$ Instituto de Ciência e Inovação em Engenharia Mecânica e Engenharia Industrial, Depar- \\ tamento de Engenharia Mecânica, Faculdade de Engenharia, Universidade do Porto, Porto, \\ Portugal,email: tavares@fe.up.pt
}

\begin{abstract}
Magnetic fluid hyperthermia (MFH) is considered a promising therapeutic technique for the treatment of cancer cells, in which magnetic nanoparticles (MNPs) with superparamagnetic behavior generate mild-temperatures under an AC magnetic field to selectively destroy the abnormal cancer cells, in detriment of the healthy ones. However, the poor heating efficiency of most NMPs and the imprecise experimental determination of the temperature field during the treatment, are two of the majors drawbacks for its clinical advance. Thus, in this work, different MNPs were developed and tested under an AC magnetic field $(\sim 1.10 \mathrm{kA} / \mathrm{m}$ and $200 \mathrm{kHz})$, and the heat generated by them was assessed by an infrared camera. The resulting thermal images were processed in MATLAB after the thermographic calibration of the infrared camera. The results show the potential to use this thermal technique for the improvement and advance of MFH as a clinical therapy.
\end{abstract}

Keywords. Thermal Imaging, Image Calibration, Image Processing.

\section{Introduction}

Magnetic fluid hyperthermia (MFH) is considered a promising therapeutic technique for cancer treatment that arise from the emerging of nanotechnology and the clinical urgency to develop new medicines, treatments and detection strategies for this disease [1]. This technique is based on the use of magnetic nanoparticles (MNPs), usually with superparamagnetic properties, to generate heat under an alternating magnetic field by the Brownian and Néel relaxation mechanisms [2], and also in the phys- 
iological intolerance of the abnormal cancer cells to temperatures in the range between 41 and $46{ }^{\circ} \mathrm{C}$ [3]. In fact, MFH has several advantages over the common used therapeutic strategies, such as the capability of MNPs to be bio-functionalized, allowing them to be used as a non-invasive imaging agent in magnetic resonance imaging (MRI) simultaneously with MFH, offering the possibility to develop an image-guided therapy system. Thus, this therapeutic technique allows the combination of two functionalities, diagnostic and therapy (also defined as theragnostic) [4]. Furthermore, although hyperthermia has been shown to cause direct damage to cancer cells when applied as single therapy, this technique can also be applied as an adjuvant to other treatments, such as radiotherapy, chemotherapy, gene therapy and immunotherapy, with minimal or no injury at all of the normal tissues [1, 4]. However, the majors drawbacks of MFH are still the poor heating efficiency of most MNPs [5], and the imprecise experimental determination of the temperature field during the treatment, which may result in insufficient heating or in over-heating with collateral damage of the healthy tissues [6]. Usually, the temperature field is measured during the treatment using fiber optic probes. Nevertheless, these captors cannot be precisely implanted in the tumor tissues and only give local temperature information [6]. Infrared thermography, based on the physical phenomenon that all bodies at temperatures above absolute zero generate heat by radiation in the infrared portion of the electromagnetic spectrum [7], can overcome the drawbacks of the fiber optic probes, through the creation of a thermal map with the temperature range of the detected infrared radiation emitted from the body (both healthy and tumor tissues), as well as from the agglomeration of MNPs.

Thus, the aim of this work was to use infrared thermography and its potentialities to assess the heat released by MNPs when subjected to an AC magnetic field, which will serve as a screening tool to evaluate the potentiality of novel synthetized MNPs to be further applied in hyperthermia. For this purpose, the thermal images acquired by the infrared camera were calibrated and processed in MATLAB (Mathworks, USA).

\section{Materials and Methods}

\subsection{Synthesis of Magnetic Nanoparticles (MNPs)}

Before the synthesis of the MNPs, solutions of $\mathrm{FeCl}_{3} \cdot 6 \mathrm{H}_{2} \mathrm{O}(134 \mathrm{mM}), \mathrm{FeCl}_{2} \cdot 4 \mathrm{H}_{2} \mathrm{O}$ $(67 \mathrm{mM})$ and $\mathrm{NH}_{4} \mathrm{OH}(1 \mathrm{M})$ were prepared in distilled and deionized water at room temperature. Samples S1 and S2 were synthetized by co-precipitation, mixing the iron precursors, under vigorous magnetic stirring, in the stoichiometric $\mathrm{Fe}^{2+}: \mathrm{Fe}^{3+}$ ratio of $1: 2$ and heating the resultant mixture until the desired temperature $\left(30\right.$ and $75{ }^{\circ} \mathrm{C}$, respectively for $\mathrm{S} 1$ and $\mathrm{S} 2$ ). Then, the basic $\mathrm{NH}_{4} \mathrm{OH}$ (1 M) solution was added dropwise until the $\mathrm{pH} \sim 10$ was reached to promote the co-precipitation of the precursors. Sample S3 was synthetized in a similar way at room temperature. However, after the MNPs co-precipitation, the black solution was transferred to a teflon-lined stainless steel high pressure batch reactor $(125 \mathrm{~mL})$ and maintained at $180{ }^{\circ} \mathrm{C}$ under its own atmosphere during $24 \mathrm{~h}$. Sample S3 was further coated with graphene oxide (GO) by 
using a simple electrostatic self-assembly process (S3@rGO), with a similar method as the one described by Wei et al. (2012) [8].

\subsection{Physical and chemical characterization of MNPs}

The crystallite phases and core sizes of the synthesized MNPs (S1, S2, S3 and S3@rGO) were analyzed by X-ray powder diffraction (XRD, PAN'alytical X'Pert PRO, Netherlands) by using the Bragg's law.

\subsection{Experimental setup}

The adequate heat release promoted by MNPs when subjected to an AC magnetic field is one of the most important key-points for the success of MFH, and thus, directly dependent on the crystalline microstructure of the MNPs, the intensity of the AC magnetic field and the method for temperature acquisition. In order to study these three points, the experimental work was divided into two main experimental setups: (i) infrared camera calibration and (ii) thermal imaging acquisition of MNPs under an AC magnetic field.

Infrared Camera Calibration. In this infrared camera calibration test, the influence of the surface material of the observed body (beakers) and solutions used as working fluids for the dispersion of MNPs was assessed. Two beakers of $100 \mathrm{~mL}$ fabricated in borosilicate glass and polypropylene (PP) polymer, with the same dimensions, $50 \mathrm{~mm}$ $\times 70 \mathrm{~mm}$ (Outer Diameter $\times$ Height) were used, each one containing $100 \mathrm{~mL}$ of the working fluid, deionized water and glycerine $70 \%$, respectively. The objects and working fluids were further heated, using a magnetic heating stirrer plate with an integrated thermocouple (C-Mag HS7, IKA, Germany), at a heating increment of 5 ${ }^{\circ} \mathrm{C}$, between 20 and $50{ }^{\circ} \mathrm{C}$. In addition, the temperature measured by the integrated thermocouple, was confirmed with a second individual thermocouple. The thermal images were acquired with the infrared camera (E30, FLIR, USA) placed at $50 \mathrm{~cm}$ of the experimental setup, and transferred to a PC for posterior analysis using MATLAB (R2015a). The experimental tests and the parameters considered in the infrared camera calibration are shown in Table 1.

Table 1. Calibration experimental tests and the parameters considered in the infrared camera.

\begin{tabular}{cccc}
\hline Calibration test & Object material & Working fluid & Temperature range \\
\hline 1 & Glass & Deionized water & $20-50{ }^{\circ} \mathrm{C}$ \\
2 & PP & Deionized water & $20-50{ }^{\circ} \mathrm{C}$ \\
3 & PP & Glycerine $70 \%$ & $20-50{ }^{\circ} \mathrm{C}$ \\
\hline
\end{tabular}

Thermal imaging acquisition of MNPs under AC magnetic field. After the calibration of the thermography method, the experimental setup for the assessment of the heat released by the synthetized MNPs was assembled as shown in Fig. 1. The AC 
magnetic field apparatus used in this work is a non-adiabatic in-house-made device, designed to work at a maximum intensity of $1.10 \mathrm{kA} / \mathrm{m}$ and with a frequency of 200 $\mathrm{kHz}$.

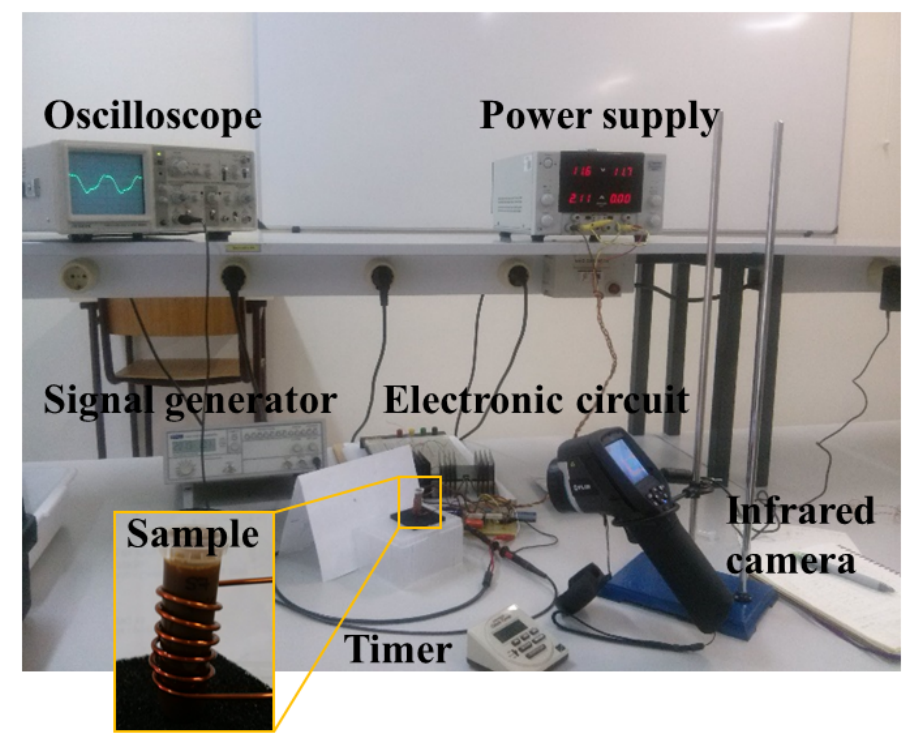

Fig. 1. Experimental setup used for the assessment of heat generated by MNPs under an AC magnetic field, monitored by an infrared camera (E30, FLIR, USA)

To investigate the influence of the working fluids in the heat released by the MNPs under an AC magnetic field, different fluids were considered to perform the infrared camera calibration (deionized water and glycerine $70 \%$ ). The infrared camera was placed at $50 \mathrm{~cm}$ of the samples, which were involved by the coil-shaped heating station (detail shown in Fig. 1), generating the AC magnetic field. The experimental assays and the parameters considered to assess the heat released by the different synthetized MNPs (S1, S2 and S3@rGO) are shown in Table 2.

Table 2. Experimental assays and the parameters considered to assess the heat generated by the different MNPs under an AC magnetic field

\begin{tabular}{ccccc}
\hline Assay & Carrier Fluid & $\begin{array}{c}\text { Density of the } \\
\text { fluid } \\
\left(\mathbf{g} / \mathbf{c m}^{\mathbf{3}}\right)\end{array}$ & $\begin{array}{c}\text { MNPs } \\
\text { Concentration } \\
(\mathbf{m g} / \mathbf{m L})\end{array}$ & $\begin{array}{c}\text { RMS } \\
\text { magnetic field } \\
(\mathbf{H})\end{array}$ \\
\hline 1 & Deionized water & 1.00 & 5.00 & $1.10 @ 200$ \\
2 & Glycerine 70\% & 1.18 & 5.00 & $1.10 @ 200$ \\
3 & Glycerine 70\% & 1.18 & 5.00 & $0.80 @ 250$ \\
\hline
\end{tabular}

The thermal imaging assays shown in Table 2 were repeated for all the synthetized MNPs (S1, S2 and S3@rGO), as well as for blank tests (working without MNPs). These blank tests allowed to check and remove the heat generated by the coil (Joule 
heating) from the global heat generated with the MNPs during the experimental tests. Periodic infrared images were taken in all thermal image assays at 0, 60, 120, 300 and $600 \mathrm{sec}$, stored in the format .JPEG and further processed in MATLAB.

\subsection{Thermal Infrared Image Processing}

The thermal infrared images acquired in the calibration and experimental tests were recorded in the format $J P E G$ with $160 \times 120$ pixels, transferred to a PC, and further converted into MATLAB readable files, with the extension .MAT, using the software ThermaCAM Researcher (trial version 2.10 Pro, FLIR, USA). This new format enables the use of the original information acquired by the infrared camera, where each pixel corresponds to the "real" captured temperature in Kelvin (K).

Infrared Camera Calibration. The thermal images acquired in the calibration tests, with extension .MAT as a matrix $120 \times 160$ corresponding to temperatures in $\mathrm{K}$, were converted to degrees Celsius $\left({ }^{\circ} \mathrm{C}\right)$ using the following code [9]:

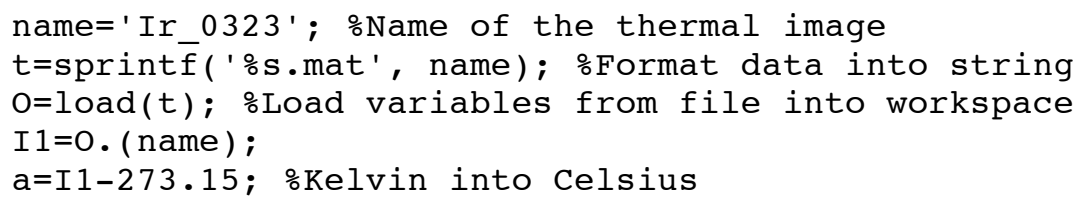

After the creation of the new matrix $(a)$, with the temperature in ${ }^{\circ} \mathrm{C}$ for each pixel, the APP Image Viewer of MATLAB was used to open it with the following command: File $>$ Import from workspace $>a>o k$. Then, by selecting the icon contrast, the image was displayed, zoomed to $200 \%$ and the desired part of the image containing the warmed fluid was selected by using the icon crop image. The region of interest was further transferred to the workspace of MATLAB using the menu File $>E x$ port To Workspace $>$ Image variable name $>I$. Finally, calling the new matrix (I) it was possible to calculate the mean temperature of this matrix, as shown in the following Matlab code line:

$>$ mean (mean (I))

Heat released by the MNPs under an AC Magnetic field. In a similar way to the image processing described above for the infrared camera calibration, the infrared images obtained in the thermal imaging assays (assays 1,2 and 3, as shown on Table 2), were transferred to MATLAB with the extension .MAT and the matrix $120 \times 160$ corresponding to temperatures in $\mathrm{K}$. The temperature was converted to a matrix in degrees ${ }^{\circ} \mathrm{C}$, using the same code above described. The new matrix $(a)$, in degrees ${ }^{\circ} \mathrm{C}$, was further open in the APP Image Viewer of MATLAB, using the following command: File $>$ Import from workspace $>a>o k$. Then, selecting the icon contrast the image was displayed, zoomed to $800 \%$ and the desired parts of the image $\left(R_{1}, R_{2}, R_{3}\right.$ and $\mathrm{R}_{4}$ ), containing the fluid heated by the MNPs under the AC magnetic field within the loops of the coil, were selected with the icon crop image, as shown in Fig. 2. 
a)

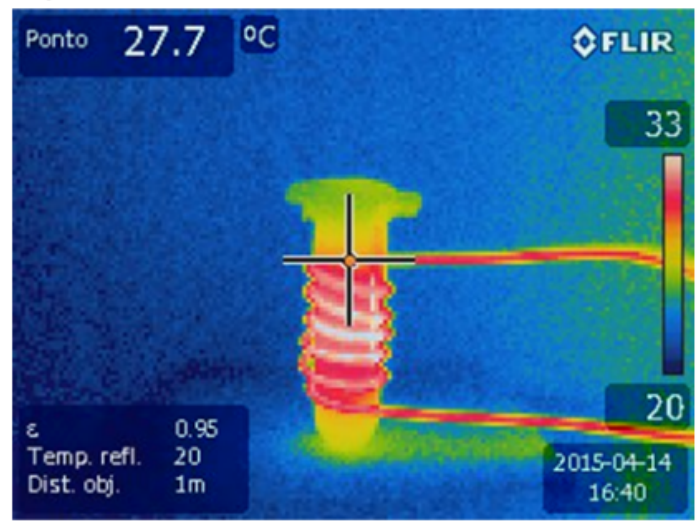

b)

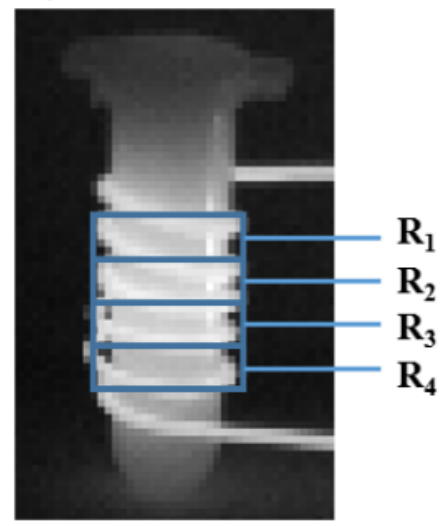

Fig. 2. Representation of an infrared image acquired in the MNPs thermal imaging assays under an AC magnetic field: a) Thermal image obtained according or the JPEG format by the infrared camera; b) Thermal imaged transformed to the .MAT format with the identification of the four regions of interest $\left(R_{1}, R_{2}, R_{3}\right.$ and $\left.R_{4}\right)$

\section{Results and Discussion}

\subsection{Physical and Chemical Characterization of MNPs}

Table 3 shows a summary of the physical and chemical characterization parameters of the synthetized MNPs (crystallite size $\left(\mathrm{d}_{\mathrm{XRD}}\right)$, lattice constant $(\mathrm{a})$ and core phases) obtained from XRD analysis.

Table 3. Physical and chemical characterization parameters obtained by XRD analysis of the synthetized MNPs.

\begin{tabular}{ccccc}
\hline Sample & $\begin{array}{c}\text { Synthesis } \\
\text { Temp. }\left({ }^{\circ} \mathrm{C}\right)\end{array}$ & $\begin{array}{c}\mathrm{d}_{\mathrm{XRD}} \\
(\mathrm{nm})\end{array}$ & $\begin{array}{c}\text { Lattice } \\
\text { constant, a }(\AA)\end{array}$ & $\begin{array}{c}\text { Core phases } \\
(\mathrm{v} / \mathrm{v})\end{array}$ \\
\hline $\mathrm{S} 1$ & $30^{\circ} \mathrm{C}$ & $12.80 \pm 0.20$ & 8.3652 & $100 \%$ Magnetite \\
$\mathrm{S} 2$ & $75^{\circ} \mathrm{C}$ & $32.20 \pm 0.30$ & 8.3770 & $100 \%$ Magnetite \\
$\mathrm{S} 3 @ \mathrm{rGO}$ & $180^{\circ} \mathrm{C}$ & $63.05 \pm 1.00$ & 5.0345 & $49.9 \%$ Hematite \\
& & $30.04 \pm 0.30$ & 8.3683 & $50.1 \%$ Magnetite \\
\hline
\end{tabular}

Table 3 puts in evidence the influence of the synthesis temperature on the crystallite sizes of the MNPs. As observed for S1 $\left(30^{\circ} \mathrm{C}\right), \mathrm{S} 2\left(75^{\circ} \mathrm{C}\right)$ and $\mathrm{S} 3\left(180^{\circ} \mathrm{C}\right)$, the increase of the synthesis temperature directly influences the growth of the magnetic core, higher crystallite sizes being obtained with higher synthesis temperatures. It is also concluded that the MNPs synthetized at $180{ }^{\circ} \mathrm{C} / 24 \mathrm{~h}$, under its own atmosphere (sample S3) not only lead to higher crystallite sizes on the magnetic core, but also 
results in the conversion of part of the magnetite composition into hematite (as observed in the lattice constant, a). It is worth mentioning that hematite is a form of iron oxide with less magnetic saturation (Ms) [10] and therefore, with lower heating power than magnetite under the same AC magnetic field.

\subsection{Infrared Camera Calibration}

As previously shown in Table 1, the infrared camera calibration was performed with three tests, where the material of the containers and the working were taken into account to determine their influence in the acquisition of the thermal images, as well as to obtain the correlation between inner and outer temperatures, Table 4 .

Table 4. Temperature results obtained by the infrared camera calibration tests regarding the temperature measurements in TC (Thermocouple temperature), FLIR (Infrared camera pointer temperature) and MAT (mean temperature of the region of interest).

\begin{tabular}{ccccccccc}
\hline \multicolumn{3}{c}{ Calibration test 1 } & \multicolumn{3}{c}{ Calibration test 2 } & \multicolumn{3}{c}{ Calibration test 3 } \\
$\begin{array}{c}\text { TC } \\
\left({ }^{\circ} \mathrm{C}\right)\end{array}$ & $\begin{array}{c}\text { FLIR } \\
\left({ }^{\circ} \mathrm{C}\right)\end{array}$ & $\begin{array}{c}\text { MAT } \\
\left({ }^{\circ} \mathrm{C}\right)\end{array}$ & $\begin{array}{c}\text { TC } \\
\left({ }^{\circ} \mathrm{C}\right)\end{array}$ & $\begin{array}{c}\text { FLIR } \\
\left({ }^{\circ} \mathrm{C}\right)\end{array}$ & $\begin{array}{c}\text { MAT } \\
\left({ }^{\circ} \mathrm{C}\right)\end{array}$ & $\begin{array}{c}\text { TC } \\
\left({ }^{\circ} \mathrm{C}\right)\end{array}$ & $\begin{array}{c}\text { FLIR } \\
\left({ }^{\circ} \mathrm{C}\right)\end{array}$ & $\begin{array}{c}\text { MAT } \\
\left({ }^{\circ} \mathrm{C}\right)\end{array}$ \\
\hline 19.3 & 18.4 & 18.7 & 20.0 & 19.6 & 20.1 & 21.7 & 21.7 & 21.7 \\
25.0 & 24.0 & 24.1 & 25.0 & 24.2 & 24.4 & 25.0 & 24.8 & 24.9 \\
30.0 & 28.3 & 28.5 & 30.0 & 29.1 & 29.6 & 30.0 & 29.5 & 29.5 \\
35.0 & 32.9 & 32.8 & 35.0 & 34.7 & 34.7 & 35.0 & 33.8 & 33.8 \\
40.0 & 37.2 & 37.1 & 40.0 & 39.3 & 39.4 & 40.0 & 38.9 & 38.7 \\
45.0 & 41.8 & 41.7 & 45.0 & 46.1 & 46.2 & 45.0 & 43.3 & 43.3 \\
50.0 & 46.3 & 46.1 & 50.0 & 49.8 & 50.1 & 50.0 & 48.8 & 48.8 \\
\hline
\end{tabular}

In general, the local temperature given by the pointer of the infrared camera are in good agreement with the mean temperature obtained from the region of interest acquired as thermal map and processed in MATLAB. The good agreement between the local and mean temperatures of the region of interest can be explained by the magnetic stirring of the liquid samples that allowed the homogenization of solutions, and thus the homogenization of the heating transfer along the thermal tests. However, Table 4 also shows a discrepancy between the temperatures recorded by the thermocouple (TC) (placed inside the fluids) and the FLIR temperatures (obtained at the outer surface of the beakers). Therefore, calibration curves and linear fits (data not shown) between the temperatures acquired by the TC and the MALTAB analysis for the three calibration tests were determined to be further used in the evaluation of the heat release caused by the MNPs under AC magnetic field tests. In addition, the results presented in Table 4 allowed the calculation of the relative errors according to:

Relative error $=\underline{\sum_{\mathrm{i}=0}^{\mathrm{n}}(\text { Inlet temperature }(\text { Thermocouple }) \text { - Outlet temperature (MATLAB) })}$ where, $n$ is the total number of measures and $i$ the measurement number. 
As a result, the relative error was found to be $2.19{ }^{\circ} \mathrm{C}$ for the calibration test $1,0.23$ ${ }^{\circ} \mathrm{C}$ for the calibration test 2 , while in the calibration test 3 an error of $0.86{ }^{\circ} \mathrm{C}$ was obtained. These results show that the use of polypropylene polymer (PP) as material of the container brings a more accurate thermal measurement between the inner and the outer surface temperatures of the observed object. Therefore, PP was found to be a better choice of material to be used in this kind of experiments. Nevertheless, the difference in the accuracy of the results obtained between the use of PP and of glass in the containers cannot be explained by the thermal conductivity of the materials. In fact, the borosilicate glass has a higher thermal conductivity than PP, being expected a priori that the difference between the inner and outer temperatures for the glass material was lower than in the PP material. Thus, a possible explanation for the results obtained, could be the interference of glass reflection in the thermal images acquisition, which in fact was observed in the experimental tests performed with this material.

\subsection{Heat Released by MNPs under AC Magnetic Field}

One of the crucial steps to perform hyperthermia tests is the colloidal stabilization of the MNPs within the working fluid, also known as ferrofluid. Therefore, in order to compare the influence of two working fluids with different densities (deionized water and glycerin $70 \%$ ) on the stabilization parameters and heat dispersion of the MNPs samples, two thermal imaging assays (thermal assays 1,2) were performed at 1.10 $\mathrm{kA} / \mathrm{m}$ and $200 \mathrm{kHz}$, during $600 \mathrm{sec}$ (cf. Table 2). In addition, thermal assay 3 was performed to check the influence of the frequency increase $(250 \mathrm{kHz})$ in the heat generated by the MNPs, Fig. 3 .

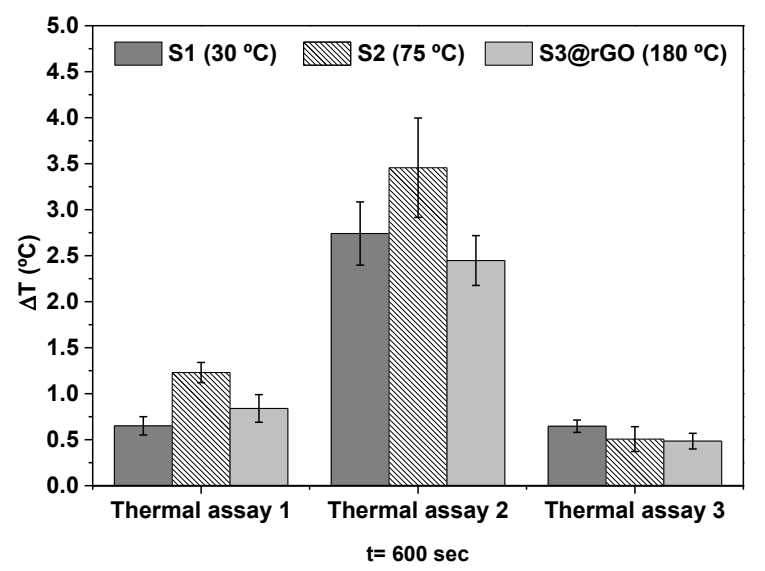

Fig. 3. Comparison of the heat released by the suspended MNPs $(5 \mathrm{mg} / \mathrm{mL})$, measured as the increment of the mean temperature of the suspensions, in different working fluids under an AC magnetic field. The mean temperatures presented are corrected by the respective calibration curves calculated from the infrared calibration tests and normalized according to the heat generated by the coil (blank tests). Error bars correspond to the standard deviation between the four regions of interest $\left(R_{1}-R_{4}\right)$. 
As shown in Fig. 3, the thermal assay 2, performed with glycerine $70 \%$ as working fluid, with $1.10 \mathrm{kA} / \mathrm{m}$ and $200 \mathrm{kHz}$, was conducted under conditions that allowed the higher heat released by synthetized MNPs, of the sample considered. Comparing the heating results of the thermal assays 1 and 2, it is also concluded that the stabilization of the MNPs in the proper working fluid is a crucial step to the performance of hyperthermia tests. The importance of this stabilization is related to the homogenization of the ferrofluid in contact with the AC magnetic field, which reach a maximum magnetic intensity within the coil. Without this stabilization, the MNPs tend to sediment in the bottom of the vials. This fact is especially critical when the AC magnetic field apparatus generate a maximum magnetic field intensity that is 20 times lower $(\sim 1.10$ $\mathrm{kA} / \mathrm{m}$, like in our case) than a commercial apparatus, which normally perform these hyperthermia tests around $20 \mathrm{kA} / \mathrm{m}$. Nevertheless, the developed apparatus used in this work allowed, with a very low AC magnetic field, a maximum temperature increase of $3.45{ }^{\circ} \mathrm{C} \pm 0.54$ for sample $\mathrm{S} 2$ (co-precipitation at $75{ }^{\circ} \mathrm{C}$ ), after $600 \mathrm{sec}$ under the AC magnetic field. In fact, this result is in good agreement with the literature, which points to the fact that MNPs under the mono-domain size and superparamagnetic properties increased their heating power as they grow in size.

For the case of the MNPs synthetized at $180{ }^{\circ} \mathrm{C}$ and coated with GO (S3@rGO), the presence of almost $50 \%$ of hematite (very lower Ms and thus, low heating power), puts these MNPs into the ferromagnetic materials. In fact, the ferromagnetic materials are considered in literature inappropriate materials to be used in biomedical application, due to the fact that these MNPs remain magnetized for a long period of time, even after the applied magnetic field is removed (high coercivity). Once more, this screening results obtained with the developed AC magnetic field are in good agreement with the characterization results and the literature in the field, resulting by this way, in a good methodology to perform screening hyperthermia characterization of MNPs.

\section{Conclusions}

Hyperthermia is a promising therapeutic technique for cancer treatment, but the clinical successful application is intrinsically linked to the development of new nanomaterials and the improvement of new methodologies to determinate the temperature fields during treatment. Therefore, in this study, MNPs with different sizes were synthetized at different synthesis temperature $\left(30,75\right.$ and $\left.180{ }^{\circ} \mathrm{C}\right)$, and its heating efficiency assessed through the use of a developed AC magnetic field apparatus and an infrared camera. The acquired thermal images were transferred to a $\mathrm{PC}$ and the images processed in MATLAB. In order to correlate the inner and outer temperatures of the containers and working fluids, several calibration tests were performed with deionized water and glycerine $70 \%$ as well as with different container materials (glass and PP). These calibration were further used in the experimental acquired data, allowing the effectively assessment of the inner temperatures achieved by the different MNPs in the different thermal assays. The screening hyperthermia assays revealed that the MNPs synthetized at $75{ }^{\circ} \mathrm{C}(\mathrm{S} 2)$ are those presenting higher heating power for hyperthermia applications, which is related to the fact that MNPs under the monodomain size and superparamagnetic properties increase their heating power as they 
grow in size. Therefore, the main conclusion of this study is that the use of infrared cameras are very effective in the assessment of the heat released by MNPs for hyperthermia applications, even when using an AC magnetic system able to create low magnetic fields.

As final remark, the good screening results obtained in this work also demonstrated the potentiality to use some of the synthetized MNPs in real hyperthermia applications. Therefore, other hyperthermia studies with commercial hyperthermia apparatus and higher magnetic intensities $(\sim 20 \mathrm{kA} / \mathrm{m})$ are now being considered in a near future using the same thermal image acquisition and processing framework (infrared camera and MATLAB), allowing the calculations of the specific absorption rate (SAR) of the MNPs, a crucial parameter in the characterization of MNPs for real magnetic hyperthermia applications.

Acknowledgments. R.O.R acknowledges the PhD scholarship SFRH/BD/97658/2013 granted by FCT - Fundação para a Ciência e a Tecnologia, in Portugal. A.M.T.S acknowledges the FCT Investigator 2013 Programme (IF/01501/2013), with financing from the European Social Fund and the Human Potential Operational Programme.

\section{References}

1. Chicheł, A., Skowronek, J., Kubaszewska, M., Kanikowski, M.: Hyperthermia - description of a method and a review of clinical applications. Rep. Prac. Oncol. Radiother. 12 (2007) 267275

2. Laurent, S., Dutz, S., Häfeli, U.O., Mahmoudi, M.: Magnetic fluid hyperthermia: Focus on superparamagnetic iron oxide nanoparticles. Adv. Colloid Interface Sci. 166 (2011) 8-23

3. Deatsch, A.E., Evans, B.A.: Heating efficiency in magnetic nanoparticle hyperthermia. J. Magn. Magn. Mater 354 (2014) 163-172

4. Ito, A., Shinkai, M., Honda, H., Kobayashi, T.: Medical application of functionalized magnetic nanoparticles. J. Biosci. Bioeng. 100 (2005) 1-11

5. Di Corato, R., Espinosa, A., Lartigue, L., Tharaud, M., Chat, S., Pellegrino, T., Ménager, C., Gazeau, F., Wilhelm, C.: Magnetic hyperthermia efficiency in the cellular environment for different nanoparticle designs. Biomaterials 35 (2014) 6400-6411

6. COST:Action TD1402 - Multifunctional Nanoparticles for Magnetic Hyperthermia and Indirect Radiation Therapy (RADIOMAG) 054/14 Brussels (2014)

7. Calin, M.A., Mologhianu, G., Savastru, R., Calin, M.R., Brailescu, C.M.: A review of the effectiveness of thermal infrared imaging in the diagnosis and monitoring of knee diseases. Infrared Physics \& Technology 69 (2015) 19-25

8. Wei, H., Yang, W., Xi, Q., Chen, X.: Preparation of Fe3O4@graphene oxide core-shell magnetic particles for use in protein adsorption. Mater. Lett. 82 (2012) 224-226

9. Bento, D.: Modelação matemática da variação da temperatura no pé. Master thesis, Polytechnic Institute of Bragança, Bragança (2011) (in Portuguese)

10. Liu, Q., Barrón, V., Torrent, J., Qin, H., Yu, Y.: The magnetism of micro-sized hematite explained. Phys. Earth Planet. Inter. 183 (2010) 387-397 